BULLETIN (New Series) OF THE

AMERICAN MATHEMATICAL SOCIETY

Volume 33, Number 3, July 1996

J-holomorphic curves and quantum cohomology, by Dusa McDuff and Dietmar Salamon, University Lecture Series, vol. 6, Amer. Math. Soc., Providence, RI, 1994, vii+207 pp., ISBN 0-8218-0332-8

The aim of this book is to present a detailed description of the theory of pseudoholomorphic 2-spheres in symplectic manifolds, with applications to symplectic invariants, quantum cohomology, and Floer homology.

In order that the reader understand the contributions of this book and the general context where they arise, I will first briefly outline the origins of symplectic geometry and its relations with almost complex geometry and pseudo-holomorphic curves. I will then explain what the Floer cohomology ring is and how it can be interpreted in the context of quantum field theory. This will naturally lead to the definition of quantum cohomology. At each step, I will describe the content of the relevant chapters of the book.

\title{
1. THE DYNAMICAL ORIGINS
}

The origins of symplectic geometry go back to the development of analytical mechanics in the eighteenth century but took a more definitive form with the Hamiltonian formulation of the laws governing the motion of a classical system. If $V$ is an $n$-dimensional manifold whose points parametrize all possible configurations of a system (here the parametrization takes into account the constraints a priori imposed on the relative positions of each constituent of the system) and if $H: T^{*} V \rightarrow \mathbf{R}$ denotes the energy associated to each configuration and momentum, the equation of the motion of a point $(q, p) \in T^{*} V$ (where $q \in V$ is the position and $p \in T^{q} V$ is the momentum) is given by:

$$
\frac{\partial H}{\partial q}(t)=p(t) \quad \frac{\partial H}{\partial p}(t)=-\dot{q}(t) .
$$

The meaning of this equation is this. Take any chart $\psi: \mathcal{U}(\subset V) \rightarrow \mathcal{O}\left(\subset \mathbf{R}^{n}\right)$ and extend it by differentiation to a chart of $d \psi: \pi^{-1}(\mathcal{U}) \rightarrow \mathcal{O} \times \mathbf{R}^{n}$ where $\pi: T^{*} V \rightarrow V$ is the projection, and write $\left(*_{\psi}\right)$ for the above system of ODEs where $(q, p)$ belongs to $\mathcal{O} \times \mathbf{R}^{n}$ and $H$ is replaced by its pullback $H \circ(d \psi)^{-1}$ on $\mathcal{O} \times \mathbf{R}^{n}$. It can be easily checked that the solutions of these systems of ODEs are independent of the choice of the chart; that is to say, the solutions of the equation $\left(*_{\psi_{1}}\right)$ coincide with the solutions of the equation $\left(*_{2}\right)$ under the identification given by $\psi_{2} \circ \psi_{1}^{-1}$. Thus the Hamiltonian equations have an intrinsic meaning. Further, they are invariant under any diffeomorphisms $\psi: T^{*} V \rightarrow T^{*} V$ induced by differentiation of a diffeomorphism of the base. Actually, the full group of diffeomorphisms which preserve these equations (for all choices of $H$ ) is the group of symplectic diffeomorphisms which is much larger than the subgroup $\operatorname{Diff}(V) \hookrightarrow \operatorname{Diff}\left(T^{*} V\right)$.

Here is a more intrinsic point of view. On $T^{*} V$ there is a canonical 1-form, the Liouville form $\lambda$ defined by $\lambda_{\alpha_{p}}(v)=\alpha_{p}(d \pi(v))$ (or by $\lambda=\sum p_{i} d q_{i}$ in a chart). Its exterior derivative $\omega=d \lambda\left(=\sum d p_{1} \wedge d q_{i}\right)$ is a closed nondegenerate 2-form. Thus it gives a pointwise pairing $T^{p} V \rightarrow T_{p} V$ at every $p \in V$, which transforms $d H$

1991 Mathematics Subject Classification. Primary 53C15; Secondary 81T20.

(C)1996 American Mathematical Society 
into a vector field $X_{H}$ (the symplectic gradient of $H$ ). In this context, Hamilton's equations simply say that the solutions are the (projection on $V$ of the) integral curves of $X_{H}$. The nondegeneracy of the form $d \lambda$ is needed for the pairing, and its closedness is needed in order that the one-parameter flow induced by $X_{H}$ (the Hamiltonian flow) preserves $d \lambda$.

This is analytic mechanics, and a great deal of the theory of ODEs is devoted to solving these equations. Fortunately, many of these equations that arise from physics or mathematics have a rich group of symmetry. It can be shown that the study of the space of solutions of such an equation can be reduced to the study of a smaller symplectic space, obtained from the initial one $T^{*} V$ by a moment map reduction that we do not need to describe here. The essential point is that, even if one is solely interested in analytical mechanics on cotangent spaces (or its quantized versions), one is naturally led to consider much more general spaces, the symplectic manifolds (that can often be compact). The simplest example is the Hamiltonian $H=\Sigma\left(q_{i}^{2}+p_{i}^{2}\right)$ on $\mathbf{C}^{n}=\mathbf{R}^{2 n}=T^{*}\left(R^{n}\right)$; since $H$ is constant along any integral curve of $X_{H}$, it is enough to consider a sphere $H^{-1}(c)$ whose moduli space of solutions is the quotient by the Hopf fibration, leading to $\mathbf{C P} \mathbf{P}^{n-1}$.

\section{The GEOMETRY OF SYMPLECTIC MANIFOLDS}

The question now is how to extract symplectic invariants from a given closed symplectic manifold? As in Riemannian geometry, these invariants are basically 2-dimensional, but Darboux's Theorem states that a symplectic form has no local invariants. Hence the (necessary) nonlocal invariants of a symplectic structure $\omega$ cannot be extracted from the integration of local invariants. A most interesting approach to defining these invariants has been introduced by Gromov in his seminal paper [7]. It is based on the following observations:

1. Up to homotopy, the space of nondegenerate 2 -forms on a given manifold is the same as the space of almost complex structures (essentially because both $\operatorname{Sp}(2 n, \mathbf{R})$ and $\operatorname{GL}(n, \mathbf{C})$ retract on their common maximal compact subgroup $U(n))$. Actually, to each symplectic form $\omega$ there corresponds a contractible (infinite-dimensional) space $\mathcal{J}(\omega)$ of all almost complex structures compatible with $\omega$, that is those $J \in$ $\operatorname{Aut}_{\mathbf{R}}(T M)$ which satisfy (a) $J_{p}^{2}=-$ id for every $p \in M$ and (b) $\omega(\cdot, J \cdot)$ is a Riemannian metric $g$ on $M$. We recover all conditions defining a Kahler manifold except the integrability of $J$. Thus symplectic geometry can be considered as the geometry of almost Kahler manifolds.

2. The real surfaces $\Sigma \rightarrow M$ which are (perhaps singular) $1_{\mathbf{C}}$-dimensional $J$ submanifolds of $(M, J)$, that is to say, those surfaces whose tangent spaces are $J$ invariant, are necessarily genuine holomorphic curves (because any almost complex structure on a real surface is automatically integrable).

3 . In the presence of the taming condition 1 above, the $g$-area of a $J$-curve $C$ in class $A \in H_{2}(M, \mathbf{Z})$ is equal to its $\omega$-area and is therefore a homological constant (like the Wirtinger inequality in Kahler geometry). This control on the energy of any $A-J$-curve (that is to say, the control at the critical Sobolev level $L^{2,1}$ ) is the essential feature of the theory: it implies, as Gromov showed, that the moduli spaces of $A-J$-curves is not necessarily compact but can be compactified in a very natural way by addition of reducible curves (that Gromov calls cusp-curves). These moduli spaces of curves behave much as in the (integrable) Kahler case. 
4. Two symplectic forms $\omega_{1}, \omega_{2}$ compatible with the same almost complex structure $J$ are isotopic through the linear path $\lambda \omega_{1}+(1-\lambda) \omega_{2}, 0 \leq \lambda \leq 1$ ! This is perhaps the most important observation of Gromov in this theory, which plays a crucial role in the classification of symplectic manifolds ${ }^{1}$. But we will not discuss this point further, since it is not directly related to the book under review.

Hence the first three conditions above suggest the following strategy. Given $(M, \omega)$, define a symplectic invariant associated to some genus $g \geq 0$ and some class $A \in H_{2}(M, \mathbf{Z})$ in the following way: for each generic $J \in \mathcal{J}(\omega)$, consider the moduli space of unparametrized $J$-curves of genus $g$ in class $A, \mathcal{M}(J, g, A)$, and define the symplectic invariant as the cobordism class of $\mathcal{M}(J, g, A)$ as $J$ varies in the connected space $\mathcal{J}(\omega)$. Of course, one must choose $g=g(A)$ so that the moduli space is of nonnegative dimension.

But there are three problems with this definition: the first one is to ensure that the space is not empty, which is a very nontrivial task that can only be solved by exhibiting at least one $J$-curve for some generic $J$ (or equivalently a $\omega$-symplectic surface in class $A)^{2}$. The second problem is due to the fact that nothing ensures in general the compactness of $\mathcal{M}(J, q, A)$, so that the cobordism would then have no meaning at all. The third problem, finally, is that even when $\mathcal{M}(J, g, A)$ is compact (or can be nicely compactified), it might frequently be null-cobordant. Although the first problem is essential, the last two can be overcome, as did Witten in [14], Ruan in [11], or Taubes in the 4-dimensional case in [13], by defining the invariant as the (signed) number of $J$-curves of genus $g(A)$ in class $A$ that meet a specified finite set of fixed representatives of homology cycles in $M$. In many cases, this number is nonzero even when $\mathcal{M}(J, g, A)$ is null-cobordant ${ }^{3}$.

The first part of the book (Chapters 1 to 5 ) gives a quite complete account of the theory of pseudo-holomorphic curves of genus 0 in symplectic manifolds, and Chapters 6 and 7 present with all details the definition and main properties of the invariants. More precisely, these seven chapters describe the local behaviour of pseudo-holomorphic curves, the Fredholm setting leading to a correct definition of the moduli spaces of $J$-curves, and a regularity criterion that ensures that a given moduli space is a nonempty smooth manifold. It then proceeds with the definition of the Gromov-Witten invariants that I briefly described above (I will come back to them below). Apart from the interest of having all this material in a single book, the main novelty of their approach is that they work in the Fréchet category of smooth maps instead of the Sobolev or Holder spaces that usually appear in this context and that were indeed used in the first book on pseudo-holomorphic curves edited by Audin-Lafontaine [3]. The fact that the Sard-Smale theorem on transversality works only in the Banach cases forces them to go back and forth between Banach and Fréchet spaces. But their point of view has a more geometric flavor and has the advantage of giving them the opportunity of making use of the

\footnotetext{
${ }^{1}$ See Lalonde-McDuff [9] for a review of the theory of $J$-curves and for its application to the classification of some symplectic 4-manifolds.

${ }^{2}$ This can be shown either by starting from a $\omega^{\prime}$-symplectic surface for some standard symplectic form $\omega^{\prime}$ which can be homotoped through symplectic forms to $\omega$, or by using spinor type arguments and Taubes's theory linking the zero set of the Seiberg-Witten equations to pseudoholomorphic curves.

${ }^{3}$ This was realised early by Gromov in [7]. He considered in this paper a special case of Ruan's definition in his study of Lagrange submanifolds; note also that the same kind of definition occurs in Donaldson's polynomials.
} 
strongest properties of pseudo-holomorphic curves (that were at that time known to hold only in the smooth category). So this approach and style will be appreciated by anyone who likes a geometric presentation; on the other hand, one should not expect from this book the optimal estimates or the statement of the theorems in the optimal Holder categories. The reader will not find either the treatment of higher genus curves (see [3] for this). But this has the pedagogical advantage of presenting the Gromov compactness theorem in its simplest version, where only the bubbling-off phenomenon can occur (since the conformal structure of the Riemann surface is automatically fixed in genus 0 ).

\section{Floer COHOMOLOGY}

The second part of the book (Chapters 8, 9, and 10) concerns the definition and properties of the quantum cohomology of symplectic manifolds and its equivalence with Floer cohomology.

Let $(M, \omega)$ be a weakly monotone $e^{4}$ symplectic manifold and $H_{t}=H_{t+1}$ a periodic Hamiltonian on $M$ of period $T=1$. Let $\psi_{1}$ be the time-one flow of the Hamiltonian. This is the symplectic transformation of $M$ which assigns to each point $p \in M$ the point $x(1)$ where $x:[0,1] \rightarrow M$ is an orbit of $H_{t}$ (i.e., satisfies $d x / d t(t)=X_{t}(x(t))$ ) with initial condition $x(0)=p$ (here $X_{t}$ is the symplectic gradient of $H_{t}$ defined by $\left.\omega\left(X_{t}, \cdot\right)=d H_{t}\right)$. Of course, the fixed points of $\psi_{1}$ coincide with the 1-periodic closed orbits of $H_{t}$. If the Hamiltonian were autonomous, the number of these fixed points would be bounded from below by the number of critical points of $H$, and actually one would be able to define the ordinary Morse complex on these points, whose homology is well known to coincide with the singular homology of $M$. Arnold conjectured in the sixties that this should continue to hold in the nonautonomous case: the lower bound on the closed orbits should still be bounded from below by the minimal number of critical points that a (Morse) function $M \rightarrow \mathbf{R}$ can have. A topological version of this conjecture was proved by Floer, who showed in [6] that, even in the nonautonomous case, one can define a complex on the closed contractible orbits of $H_{t}$ whose homology coincides - as a graded group - with the ordinary homology of $M$ (at least when $\pi_{2}(M)=0$ ). The idea is to replace points of $M$ by contractible loops and the usual Morse theory of the autonomous case by the infinite-dimensional Morse theory of the action functional (roughly, the key idea is to apply to this problem what string theory does to quantum mechanics: replace points by closed strings and change correspondingly the Lagrangian and the action).

So we are interested in constructing a cohomology whose generators are the $T$ periodic contractible closed orbits of $H_{t}$. If $x$ is such a closed contractible orbit bounding two discs $u_{1}, u_{2}: D^{2} \rightarrow M$, let's say that $\left(x, u_{1}\right)$ is equivalent to $\left(x, u_{2}\right)$ if $u_{1} \#\left(-u_{2}\right)$ is homologous to zero. Denote simply by $\tilde{x}$ such a class and by $\widetilde{\mathcal{L} M}$ the space of all these classes (on which the spherical 2-classes of $H_{2}(M)$ obviously act). On this space is defined the following symplectic action functional:

$$
a_{H}(x, u)=\int_{B} u^{*} \omega+\int_{0}^{1} H_{t}(x(t)) d t
$$

\footnotetext{
${ }^{4} \mathrm{~A}$ monotone symplectic manifold obeys $c_{1}=\lambda[\omega]$ for some nonnegative real number $\lambda$, where $c_{1}$ is the first Chern class of the tangent bundle of $(M, \omega)$. The condition of weak monotonicity is a technical generalization of monotonicity that ensures that the $J$-curves in $(M, \omega, J)$ are well behaved.
} 
which satisfies $a_{H}(A \# \tilde{x})=a_{H}(\tilde{x})+\omega(A)$ for any spherical class $A \in H_{2}(M)$. Thus the functional is defined on the covering $\widetilde{\mathcal{L} M}$ of the loop space $\mathcal{L} M$. On this latter space, the action functional is a closed 1-form: thus Morse theory in this setting is precisely an infinite-dimensional version of the Novikov homology associated to a closed 1-form on some manifold. It is easy to see that the critical points of $a_{H}$ on $\widetilde{\mathcal{L M}}$ are the points $\tilde{x}=(x, u)$ with $x$ a closed 1-periodic orbit of $H_{t}$. These points are graded by the Conley-Zehnder index which is computed by trivialising the tangent bundle of $M$ over the disc $u(B)$ and considering the path of symplectic matrices generated by the linearized Hamiltonian flow around $x(t)$. One can then define the Floer-Novikov complex by $C^{k}(H)=\sum_{\operatorname{ind}(\tilde{x})=k} c_{\tilde{x}} \tilde{x}$. In order to avoid problems with noncompacity of the moduli space of gradient flowlines of $a_{H}$, we also require that, for any $c \in R$, the latter sum contains only a finite number of $\tilde{x}$ with $a_{H}(\tilde{x}) \leq c$. Thus this complex is a module over the Novikov ring $\Lambda=\Lambda_{\omega}$ defined in the following way: let $\Gamma \subset H_{2}(M)$ be the image of the Hurewicz homomorphism, and define $\Lambda$ as a kind of completion of the group ring of $\Gamma$. It is the ring of all sums with, say, integer coefficients

$$
\lambda=\sum_{A \in \Gamma} \lambda_{A} e^{2 \pi i A}
$$

such that for any $c$ there are only finitely many nonzero terms with $\omega(A) \leq c$. This ring carries a natural grading given by $\operatorname{deg}\left(e^{2 \pi i A}\right)=2 c_{1}(A)$. The choice of the grading is dictated by the fact that $\operatorname{ind}(A \# \tilde{x})=\operatorname{ind}(\tilde{x})+2 c_{1}(A)$.

Now consider the upward gradient flowlines of $a_{H}$ with respect to a $L^{2}$-metric on $\widetilde{\mathcal{L} M}$ induced by an almost complex structure on $M$ (which together with $\omega$ defines an almost Kahler structure on $M$ ). They are given by solutions $v: \mathbf{R} \times \mathbf{R} / \mathbf{Z} \rightarrow M$ of the elliptic PDE $\bar{\partial}_{J}(v)=\nabla H_{t}(v)$ whose ends converge to closed orbits $\tilde{x}^{-}, \tilde{x}^{+}$ with the disc $u^{+}$obtained from the disc $u^{-}$by gluing the cylinder $v$. When the indices of $\tilde{x}^{-}, \tilde{x}^{+}$differ by 1 , the space of these flowlines, once quotiented out by the time shift, is a compact 0 -dimensional manifold. One can therefore define the coboundary operator of the complex $C^{*}(H)$ in the usual way in Morse theory, that is, by $\delta \tilde{x}=\sum_{\operatorname{ind} \tilde{y}=\operatorname{ind} \tilde{x}+1} \eta(\tilde{x}, \tilde{y}) \tilde{y}$ where $\eta(\tilde{x}, \tilde{y})$ counts the gradient flowlines from $\tilde{x}$ to $\tilde{y}$. This complex gives rise to the Floer cohomology $H F^{*}\left(M, \omega, H_{t}, J\right)$. Floer [6] (and Hofer-Salamon [8] in this general setting) showed that this cohomology is actually independent of the choice of $H_{t}$ and $J$. Moreover:

Theorem 3.1 (Hofer-Salamon [8]). If either $M$ is monotone, or $c_{1}(A)=0$ for all $A \in \Gamma$, or the minimal Chern number is greater or equal to half the real dimension of $M$, then $H^{*}\left(M, \omega, H_{t}, J\right)$ is isomorphic, as graded groups (with rational coefficients $)$, to $H^{*}(M) \otimes \Lambda_{\omega}$.

In particular, $H F^{*}$ is isomorphic to $H^{*}$ if $\pi_{2}(M)=0$.

All this is very nicely presented in the last chapter (Chapter 10) of McDuffSalamon's book, which concludes with the definition of the ring structure on Floer cohomology. This definition is quite natural: the map

$$
C^{k}(H) \times C^{l}(H) \rightarrow C^{k+l}(H)
$$

sends $\left(\tilde{x}_{1}, \tilde{x}_{2}\right)$ to $\tilde{x}_{1} \star \tilde{x}_{2}=\sum \alpha_{\tilde{x}} \tilde{x}$ where $\alpha_{\tilde{x}}$ counts the number of elements in the moduli space $\mathcal{M}\left(\tilde{x}_{1}, \tilde{x}_{2}, \tilde{x}_{3}\right)$ consisting of spheres $S$ with three punctures presented as flat cylindrical ends, which are $J$-holomorphic outside the ends, satisfy the gradient flowline equation on each end (more precisely, one replaces $H$ by $\tilde{H}$ defined 
on the product $S \times M$ with a bump function in order to smooth out each $\widetilde{H}=H$ (defined on each end) to 0 where it approaches the compact part of $S$ ), and converge to $\tilde{x}_{1}, \tilde{x}_{2}, \tilde{x}_{3}$ on the ends.

The associativity $\left(\tilde{x}_{1} \star \tilde{x}_{2}\right) \star \tilde{x}_{3}=\tilde{x}_{1} \star\left(\tilde{x}_{2} \star \tilde{x}_{3}\right)$ is now obvious: the left-hand side is obtained by gluing two spheres with three cylindrical ends along a long neck; this procedure cancels out one hand on each sphere. The right-hand side is obtained similarly. In both cases we get spheres with four punctures as the result of the gluing. To prove that they both lead to the same counting in moduli spaces, one deforms the conformal structure of the first sphere with four punctures to the conformal structure of the second. Therefore, under the hypotheses of the above theorem, we get a ring structure on $H F^{*}(M, \omega)=H^{*}(M) \otimes \Lambda_{\omega}$.

The end of Chapter 10 gives a sketch of an argument showing that this ring coincides with the so-called quantum cohomology of $M$.

\section{Quantum field theory and the Atiyah-Witten point of VieW ON FLOER HOMOLOGY}

As I mentioned above, the central chapters of the book (Chapters 6, 7, 8, and 9) are devoted to a mathematical definition of the Gromov-Witten invariants and of quantum cohomology.

Since I have already described an alternate definition of quantum cohomology (via Floer cohomology), I begin by explaining briefly the motivation, arising from quantum field theory, that led Witten in [15] to the definition of the invariants entering in the definition of quantum cohomology (I will come back below to the mathematical definition given by McDuff and Salamon, based on works by Ruan and Tian [12]).

The "physical" definition of quantum cohomology was in some sense motivated by Atiyah's suggestion in [1] that Floer theory can be interpreted as a quantum field theory, either in the context of string theory or of the (nonlinear) sigma model. This program was realised by Witten in [14], and this led him to introduce the quantum cohomology in [15]. So first, let's briefly recall what a nonrelativistic QFT should be, following Feynman's approach (see [5]).

A classical mechanical theory is determined by the choice of a Lagrangian on $T V$ or of a Hamiltonian on $T^{*} V$, where $V$ is a manifold, the configuration space. A classical field theory is defined in a similar way by the choice of a bundle over $V$ whose sections are the fields $\phi$, and by the choice of a Lagrangian $L(t, q, \phi(q), \phi(q))$ whose integral for a given path $\phi_{t}=\phi(t, \cdot)$ is the action. In both cases, the dynamics (or time evolution) is given by following the paths which are critical points of the action. A quantum mechanical theory assigns to $V$ the space $L^{2}(V)$ of wave functions $\psi(q)$ with complex values. Its dynamics $\psi_{t}(q)=\psi(t, q)$ is determined by the following integral equation:

$$
\psi\left(t^{\prime}, q^{\prime}\right)=\int_{V} K\left(q^{\prime}, q ; t^{\prime}-t\right) \psi(t, q) d q
$$

assuming that an initial state $\psi(t, q)$ is given. Here the propagation kernel $K$ reflects the density of probability that the particle be at position $q^{\prime}$ at time $t^{\prime}$ given that it was at $q$ at time $t$. This kernel must satisfy the initial condition $\lim _{t \rightarrow 0} K\left(q^{\prime}, q ; t\right)=\delta\left(q^{\prime}-q\right)$ and the composition law

$$
K\left(q^{\prime \prime}, q ; t^{\prime \prime}-t\right)=\int K\left(q^{\prime \prime}, q^{\prime} ; t^{\prime \prime}-t^{\prime}\right) K\left(q^{\prime}, q ; t^{\prime}-t\right) d q^{\prime} .
$$


By iterations of this formula as $\Delta t \rightarrow 0$, and based on considerations of Dirac, Feynman in [5] concludes that the kernel has the following functional integral representation:

$$
K\left(q^{\prime}, q ; t^{\prime}-t\right)=\int_{\mathcal{C}} e^{2 \pi i \mathcal{S} / h} D(q(t))
$$

where the integral is taken over the space $\mathcal{C}$ of all paths from $q, t$ to $q^{\prime}, t^{\prime}$ and where $S=\int L$ is the action of each path. This is the Feynman "sum-over-histories" formulation of the dynamical law of a system with a finite number of degrees of freedom.

The same quantization applies to field theory: here the unknown is the wave function $\Psi_{t}(\phi)$ defined on the space of fields, and the same considerations lead to a similar formula for the propagation kernel (or "partition function"):

$$
K\left(\phi^{\prime}, \phi ; t^{\prime}-t\right)=\int_{\mathcal{C}} e^{2 \pi i \mathcal{S} / h} D(\phi(t)),
$$

but this time $\mathcal{C}$ consists of paths on the space of fields. Hence the quantum dynamics is determined by an integral over the space of classical fields (or points).

More generally, an intrinsic $d+1$-quantum field theory associates to a $d$-dimensional manifold $W$ a complex vector space $\mathcal{H}(W)$, to a manifold $V$ with $\partial V=$ $W$ an element $Z(V) \in \mathcal{H}(W)$, in such a way that (1) $\mathcal{H}\left(W_{1} \coprod W_{2}\right)=\mathcal{H}\left(W_{1}\right) \otimes$ $\mathcal{H}\left(W_{2}\right)$, (2) $\mathcal{H}(-W)=\mathcal{H}(W)^{*}$, and (3) the following composition law holds: if $V=V_{1} \cup_{W} V_{2}$ where $\partial V_{1}=W_{1} \amalg W$ and $\partial V_{2}=-W \amalg W_{2}$, then $Z(V)=Z\left(V_{1}\right) \circ$ $Z\left(V_{2}\right): \mathcal{H}\left(W_{1}\right) \rightarrow \mathcal{H}\left(W_{2}\right)$. The fields on $V$ are functions, sections of a bundle, or maps to a manifold $M$ (in which case the space of fields is not a vector space: then the theory is called a nonlinear sigma model). Finally the action $\mathcal{S}(\phi)$ is the integral over $V$ of some Lagrangian depending on $\phi$ and its derivatives. The (classical) observables are complex valued functions on the space of fields.

The partition function (or propagation kernel when there is a time direction) is as above

$$
Z(M)=\int_{\phi^{\prime} s} e^{2 \pi i c \mathcal{S}(\phi)} D \phi
$$

where $1 / c$ is a coupling constant. The correlation value of the observables $\mathcal{O}_{1}, \ldots, \mathcal{O}_{k}$ is by definition

$$
\left\langle\mathcal{O}_{1} \cdots \mathcal{O}_{k}\right\rangle=\int_{\phi^{\prime} s} e^{2 \pi i c \mathcal{S}(\phi)} \mathcal{O}_{1} \ldots \mathcal{O}_{k} D \phi
$$

which are, as above, expressed uniquely in terms of classical fields. The theory is topological if the partition function and the observables do not depend on the choice of the metrics on the manifolds involved.

Now Witten in [14] (but see also Baulieu-Singer [4]) considers the topological sigma model with $V$ equal to a Riemann surface $\Sigma$ (the Riemann sphere for instance), $M$ a symplectic manifold, and the fields being the triplets $(\phi, \chi, \rho)$ where $\phi$ is a smooth map from $\Sigma$ to $M$, and $\chi, \rho \in \Omega^{0}\left(\Sigma, \phi^{*} T M\right), \Omega^{1}\left(\Sigma, \phi^{*} T M\right)$. His Langrangian leads to an action which essentially coincides with the energy ${ }^{5}$. Witten shows further that, essentially, the only global observables are those of the form $\mathcal{O}_{\alpha}(z)$, where $z \in \Sigma$ and $\alpha$ is a closed differential form: it is zero except if the map $\phi$ sends $z$ to the Poincare dual of $\alpha$ (in which case it is equal to the intersection number). Physical considerations (BRST-invariance) suggest that the correlation

\footnotetext{
${ }^{5}$ One might look at this from the point of view of string (field) theory: see the book [16] edited by Yau, and in particular the first four papers.
} 
functions $\left\langle\mathcal{O}_{\alpha_{1}}\left(z_{1}\right) \cdots \mathcal{O}_{\alpha_{k}}\left(z_{k}\right)\right\rangle$ are topological invariants in the sense that they are invariant under continuous change of $\omega, J$, and change of the $k$ points on $\Sigma$. In order to evaluate ${ }^{6}$

$$
\left\langle\mathcal{O}_{\alpha_{1}}\left(z_{1}\right) \cdots \mathcal{O}_{\alpha_{k}}\left(z_{k}\right)\right\rangle=\int_{\phi, \chi, \rho} e^{-\mathcal{L}(\phi, \chi, \rho)} \mathcal{O}_{\alpha_{1}}\left(z_{1}\right) \cdots \mathcal{O}_{\alpha_{k}}\left(z_{k}\right) D \phi
$$

Witten uses the invariance of the expression with respect to changes in the metric: changing $g$ to $c g$ for arbitrarily large $c$ produces a similar change in $\mathcal{L}$ which becomes $c \mathcal{L}$. Thus the above Feynman integral is dominated by fields which are minima of $\mathcal{L}$. These minima are easily seen to coincide with $J$-holomorphic maps $\Sigma \rightarrow M$. Hence, the integral reduces to the sum (counting multiplicities) of all rational $J$ curves which send the marked points $z_{1}, \ldots, z_{k}$ to representants of the Poincaré duals of $\alpha_{1}, \ldots, \alpha_{k}{ }^{7}$. This sum splits into sums related to given homology classes of $J$-rational curves, so that finally one is led to define the Gromov-Witten invariant $\Psi_{A, k}\left(\alpha_{1}, \ldots, \alpha_{k}\right)$ as the number of rational $J$-curves in class $A$ that send the fixed marked points to the given representatives of the Poincaré duals of the $\alpha_{i}^{\prime} s$.

The reader will find in Chapters 6 and 7 of McDuff-Salamon's book a rigorous and concise definition of this invariant, as well as the definition of another similar invariant that they denote $\Phi_{A, k}\left(\alpha_{1}, \ldots, \alpha_{k}\right)$ which counts the number of unparametrized rational curves in class $A$ which meet each of the given representants of the Poincaré dual of the forms $\alpha_{i}$. This indeed requires some care, since one has to "count" the rational curves with sign and multiplicities for "generic" data. Their presentation of the subject is inspired from Ruan-Tian's work in [12]. Note that the invariants are nonzero only when the dimension of the moduli space of $J$-A-rational curves (as given by the Atiyah-Singer index theorem) and the dimensions of the cycles $P D\left(\alpha_{i}\right)$ are properly chosen.

\section{Quantum COHOMOLOGY}

Finally, Chapters 8 and 9 of the book give a mathematical presentation of quantum cohomology. This cohomology was first introduced by Witten in [15] as a particular case of the fact that the correlation functions (which define the above Gromov-Witten invariants) must satisfy the composition law. Here is the definition of this cohomology, as given by McDuff and Salamon.

Define $Q H^{*}(M)=H^{*}(M) \otimes \Lambda$ where $\Lambda$ is the Novikov ring defined above and $H^{k}(M)$ is $H^{k}(M, \mathbf{Z})$ mod its torsion subgroup. It is graded by

$$
Q H^{k}(M)=\bigoplus_{j=0}^{2 n} H_{j}(M) \otimes \Lambda_{k-j},
$$

and thus any class there can be written as $\sum_{A} \alpha_{A} e^{2 \pi i A}$, for $\alpha_{A} \in H^{k-2 c_{1}(A)}(M)$. Note that $Q H^{*}(M)$ is a module over $\Lambda$ in an obvious way. The cup product

$$
Q H^{k}(M) \otimes Q H^{l}(M) \rightarrow Q H^{k+l}(M)
$$

\footnotetext{
${ }^{6}$ In this formula, $\sqrt{-1}$ does not appear in the exponential, but this is in the spirit of this kind of computation where one usually extends the value of the integral to complex values of the coupling constant.

${ }^{7}$ Here the invariance of the correlation function with respect to the change $\mathcal{L} \mapsto c \mathcal{L}$ makes the computation quite easy; however, in general, a similar method in computing Feynman integrals, the stationary phase approximation, leads to a more complicated computation.
} 
is defined by

$$
\alpha * \beta=\sum_{A}(\alpha * \beta)_{A} e^{2 \pi i A}
$$

where $(\alpha * \beta)_{A} \in H^{k+l-2 c_{1}(A)}(M)$ is given by

$$
\int_{c}(\alpha * \beta)_{A}=\Phi_{A}(\alpha, \beta, \gamma)
$$

for $c \in H_{k+l-2 c_{1}(A)}(M)$ and $\gamma$ equal to the Poincaré dual of $c$.

The authors establish the following theorem as in [12], but with a new argument for the proof of the associativity:

Theorem 5.1. Assume that $(M, \omega)$ is weakly monotone. Then the cup product on $Q H^{*}(M)$ is associative, distributive, and skew-commutative.

Recently, Piunikhin, Salamon, and Schwarz [10] have showed that this ring structure on $Q H^{*}(M)$ does agree with the ring structure coming from the pair-of-pant product on the Floer cohomology. As mentioned above, the book by McDuff and Salamon contains a sketch of this proof at the end of the last chapter.

Note that, in the definition of the quantum product, the contribution of the class $A=0$ gives the ordinary cup product structure of $M$. Thus, morally, the quantum product is a deformation due to the presence of nontrivial holomorphic 2 -spheres.

\section{Concluding Remarks}

This book is nicely written, very timely, and technically correct. Two appendices that cover the technical aspects of the analysis involved in the study of holomorphic 2-spheres have been added at the end of the book. Chapter 8 contains a lot of intriguing relations between quantum cohomology and other subjects such as the Verlinde algebra, the Givental homological geometry, Frobenius manifolds, and integrable systems. Chapter 8 also contains the computation of the quantum cohomology ring of some manifolds due to various authors. The only criticism that I would make is that the book seems sometimes a bit too eclectic: there is not much place for explaining the relationships between the various subjects treated (but this is normal, since much of the content of Chapter 8 is the result of very recent research) and there is no motivation from physics. I hope that this review can help fill that gap, at least partially.

I am very grateful to Lisa Jeffrey for illuminating discussions on some parts of the dictionary between physics and geometry. I also thank John Harnad for a discussion on the BRST-invariance. I mention, finally, the recent article [2] (in French) by Audin in the 1995-96 Bourbaki Seminar, entitled "Cohomologie quantique", which contains a quite complete review of the mathematical aspects of the subject.

\section{REFERENCES}

1. M. Atiyah, New invariants of three and four-dimensional manifolds, The Mathematical Heritage of H. Weyl (R. Wells, ed.), Proc. Sympos. Pure Math., vol. 48, Amer. Math. Soc., Providence, RI, 1988. MR 89m:57034

2. M. Audin, Cohomologie quantique, Séminaire Bourbaki, no. 806, 1995-96.

3. M. Audin and J. Lafontaine (eds.), Holomorphic curves in symplectic geometry, Progress in Math., vol. 117, Birkhäuser, Basel and Boston, MA, 1994. MR 95i:58005

4. L. Baulieu and I. M. Singer, The topological sigma model, Commun. Math. Phys. 125 (1989), 227-237. MR 91b:81153 
5. R. P. Feynman and A. R. Hibbs, Quantum mechanics and paths integrals, McGraw-Hill, New York, 1965.

6. A. Floer, Symplectic fixed points and holomorphic spheres, Commun. Math. Phys. 120 (1989), 575-611. MR 90e:58047

7. M. Gromov, Pseudo-holomorphic curves in symplectic manifolds, Invent. Math. 82 (1985), 307-347. MR 87j:53053

8. H. Hofer and D. Salamon, Floer homology and Novikov rings, preprint 1993.

9. F. Lalonde and D. McDuff, $J$-curves and the classification of rational and ruled symplectic 4-manifolds (Proc. Symplectic Topology Program, Newton Institute), Cambridge Univ. Press, to appear.

10. S. Piunikhin, D. Salamon, and M. Schwarz, Symplectic Floer-Donaldson theory and quantum cohomology, preprint 1995.

11. Y. Ruan, Topological sigma model and Donaldson type invariants in Gromov theory, preprint 1993.

12. Y. Ruan and G. Tian, A mathematical theory of quantum cohomology, J. Differential Geom., to appear.

13. C. Taubes, The Seiberg-Witten and the Gromov invariants, preprint 1995.

14. E. Witten, Topological sigma models, Commun. Math. Phys. 118 (1988), 411-449. MR 90b: 81080

15. _ Two-dimensional gravity and intersection theory on moduli space, Surveys Differential Geom. 1 (1991), 243-310. MR 93e:32028

16. S. T. Yau (ed.), Mathematical aspects of string theory, Adv. Ser. Math. Phys., vol. 1, World Scientific, Singapore, 1986. MR 89m:81001

FRANÇOIS LALONDE

Université du QuéBec À MontréAL E-mail address: flalonde@math.uqam.ca

AND

Centre de Recherches Mathématiques (Université de Montréal)

E-mail address: lalonde@crm.umontreal.ca 